\title{
Interleukin-6 level is an independent predictor of right ventricular systolic dysfunction in patients hospitalized with COVID-19
}

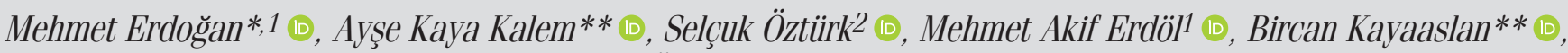 \\ Yunus Emre Özbebek* (D), Rahmet Güner** (1) \\ Departments of *Cardiology, and **Infectious Disease and Clinical Microbiology, Faculty of Medicine, \\ Ankara Yıldırım Beyazit University; Ankara-Turkey \\ ${ }^{1}$ Department of Cardiology, Ministry of Health, Ankara City Hospital; Ankara-Turkey \\ ${ }^{2}$ Department of Cardiology, Faculty of Medicine, Yozgat Bozok University; Yozgat-Turkey
}

\section{ABSTRACT}

Objective: Cytokine storm with elevated levels of multiple proinflammatory cytokines and inflammatory system activation underlie the pathogenesis of coronavirus disease 2019 (COVID-19). In this study, we aimed to investigate whether increased interleukin (IL)-6 levels can predict right ventricular (RV) systolic impairment in patients hospitalized with COVID-19.

Methods: This prospective, observational study included 100 consecutive patients hospitalized with mild and moderate COVID-19. All the patients underwent chest computerized tomography, detailed laboratory tests including IL-6, and two dimensional (2D) transthoracic echocardiography (TTE) with assessment of 2D conventional and Doppler echocardiography parameters and RV systolic functions.

Results: After the elimination of six patients with exclusion criteria, the remaining patients were classified into two groups, namely normal RV systolic functions $(n=60)$ and impaired RV systolic functions $(n=34)$. IL-6 levels were significantly higher in patients with impaired RV systolic functions than in those with normal RV systolic functions $(20.3,4.6, p<0.001$, respectively). Tricuspid annular plane systolic excursion and RV derived tissue Doppler imaging (TDI) S' measurements were similar between the two groups. RV fractional area change was significantly lower, and RV TDI derived index of myocardial performance was significantly higher in patients with impaired RV systolic functions. In multivariate analysis, IL-6 levels independently predicted deterioration in RV systolic function at a significant level (odds ratio: 1.12, 95\% confidence interval: $1.04-1.20, p=0.003)$.

Conclusion: IL-6 is an independent predictor of RV systolic impairment in patients hospitalized with mild and moderate COVID-19 suggesting a possible pathogenetic mechanism. IL-6 levels can be used to predict RV systolic impairment in patients suffering from this infection.

Keywords: coronavirus disease 2019, interleukin-6, transthoracic echocardiography, right ventricle

Cite this article as: Erdoğan M, Kaya Kalem A, Öztürk S, Erdöl MA, Kayaaslan B, Özbebek YE, et al. Interleukin-6 level is an independent predictor of right ventricular systolic dysfunction in patients hospitalized with COVID-19. Anatol J Cardiol 2021; 25: 555-64.

\section{Introduction}

Coronavirus disease 2019 (COVID-19), which is the most recent viral pandemic of $21^{\text {th }}$ century, has become a global public health problem. It is caused by a novel coronavirus called the severe acute respiratory syndrome coronavirus 2 (SARS-CoV-2). The clinical spectrum of COVID-19 varies from asymptomatic infection to respiratory illness encompassing septic shock, multiple organ failure, and acute respiratory distress syndrome (ARDS), and subsequently death $(1,2)$. ARDS is the most severe form of the disease and associated with prolonged ventilation times and increased short-term mortality in mechanically ventilated patients with COVID-19 (3). In addition, right ventricular (RV) functions are significantly impaired in patients with ARDS

Address for Correspondence: Dr. Mehmet Erdoğan, Ankara Yıldırım Beyazıt Üniversitesi Tıp Fakültesi, Kardiyoloji Anabilim Dalı, Ankara-Türkiye

Phone: +90 3125526000 E-mail: mhmterdogan@windowslive.com

Accepted Date: 10.03.2021 Available Online Date: 02.07.2021

(C) Copyright 2021 by Turkish Society of Cardiology - Available online at www.anatoljcardiol.com DOI:10.5152/AnatolJCardiol.2021.24946 


\section{HIGHLIGHTS}

- Interleukin (IL) 6 is an acute phase reactant that plays a significant role in the pathogenesis of coronavirus disease 2019 (COVID-19).

- IL-6 levels were significantly higher in patients with impaired right ventricle (RV) systolic functions than in patients with normal RV systolic functions who were hospitalized with mild or moderate COVID-19.

- IL-6 levels independently predicted RV systolic impairment in patients hospitalized with mild or moderate COVID-19.

because of its anatomical and physiological relationship with the respiratory system and supposed to be related with poor outcomes in this high-risk patient group (4).

A cytokine storm with elevated levels of multiple proinflammatory cytokines and inflammatory system activation underlie the pathogenesis of SARS-CoV-2 infection. Thus, increased C-reactive protein (CRP), ferritin, and interleukin (IL) 6 levels emerged as significant biomarkers of the disease (5). Moreover, previous studies indicate an independent association between IL- 6 levels and RV dilatation and functions $(6,7)$. To the best of our knowledge, there is no study in the literature investigating the relationship between RV functions and IL-6 levels in patients with COVID-19. Accordingly, in this study, we sought to investigate whether increased IL-6 levels can predict RV systolic impairment in patients hospitalized with mild and moderate COVID-19.

\section{Methods}

\section{Study protocol and participants}

The population of this prospective and observational study encompassed 100 consecutive patients with COVID-19 hospitalized at our tertiary center hospital between May and June 2020. COVID-19 was diagnosed according to the World Health Organization interim guidance report (8). Viral infection with SARS-CoV-2 was detected through RNA analysis in throat swap samples using real-time reverse transcription-polymerase chain reaction (Beijing Genomics Institution and Geneodx Biotechnology Co. Ltd.). In addition, multidetector computerized tomography (CT) was performed in all the hospitalized patients to examine their lungs. The discharge criteria from hospital were nonexistence of fever for at least three days, clinical amelioration of symptoms, and two negative results of SARS-CoV-2 RNA obtained at least at 24-hour intervals. All procedures applied in this study were compatible with the Declaration of Helsinki, and written informed consent was provided by all the participants. In addition, the Local Ethics Committee and Turkish Ministry of Health approved the study protocol (E1-20-611/21-05-2020).

Exclusion criteria were defined as follows; history of heart failure $(n=1)$, moderate/severe valvular heart disease and/or prosthetic heart valve $(n=2)$, atrial fibrillation $(n=2)$, cardiomyopathy, dextrocardia $(n=1)$, history of primary pulmonary hypertension, and myocarditis. Patients without evidence of CT findings of pneumonia were classified as mild, whereas patients with radiological evidence of pneumonia were classified as moderate according to the clinical classification system developed by National Health Commission \& National Administration of Traditional Chinese Medicine (9). Patients with severe or critical condition or patients followed with invasive mechanical ventilation before the enrollment were not included in the study. The severity of the infection worsened in seven patients during the hospital follow-up period, and these patients were transferred to the intensive care unit. One patient was hospitalized again for re-infection after discharge. However, they were not excluded from the final analysis, and none of the study patients died during the hospital stay.

\section{Laboratory procedures}

Peripheral venous blood samples were obtained from the large antecubital vein at the time of admission and during clinical visits. In addition to total complete blood count and biochemical tests, CRP, D-dimer, procalcitonin, ferritin, high-sensitive troponin-I (hs-Tnl), and IL-6 levels were determined. Blood samples were taken into standardized ethylenediamine tetraacetic acid containing tubes for total complete blood count, and measurements were performed immediately after the blood sampling.

Total complete blood count (Symex K-1000, Kobe, Japan) and biochemical analyzes (Roche Diagnostic Modular Systems, Tokyo, Japan) were performed at the biochemistry laboratory of our hospital. Albumin, sodium, blood urea nitrogen, creatinine, aspartate aminotransferase, alanine aminotransferase, and lipid profile were measured through standard methods. Serum CRP levels were detected through the immune nephelometric method (NFL BN-II; Dade Behring, Siemens). D-dimer levels were determined quantitatively using a Sysmex CS-5100 hemagglutinin analyzer. Procalcitonin levels were determined using a Biomerieux Mini VIDAS automatic fluorescence immunoanalyzer. Serum ferritin and IL-6 levels were detected using the electrochemiluminescence method (Cobas E601, Roche), and hs-Tnl levels were determined using an Elecsys 2010 analyzer (Roche Diagnostics, Basel, Switzerland).

\section{Transthoracic echocardiography}

All the study patients underwent detailed two-dimensional (2D) and Doppler echocardiographic examinations performed by two experienced research echocardiographers blinded to the clinical status and laboratory data of the patients. Examinations were performed by using a commercially available echocardiograph equipped with a 2-4 MHz transducer with Philips Affiniti 50 (Philips Healthcare, Andover, Netherlands) in a dedicated echocardiography laboratory for the examination of patients with COVID-19 during the pandemic. All the examinations were performed in the left lateral decubitus position, adhering to a focused, time-efficient protocol with use of protective equip- 


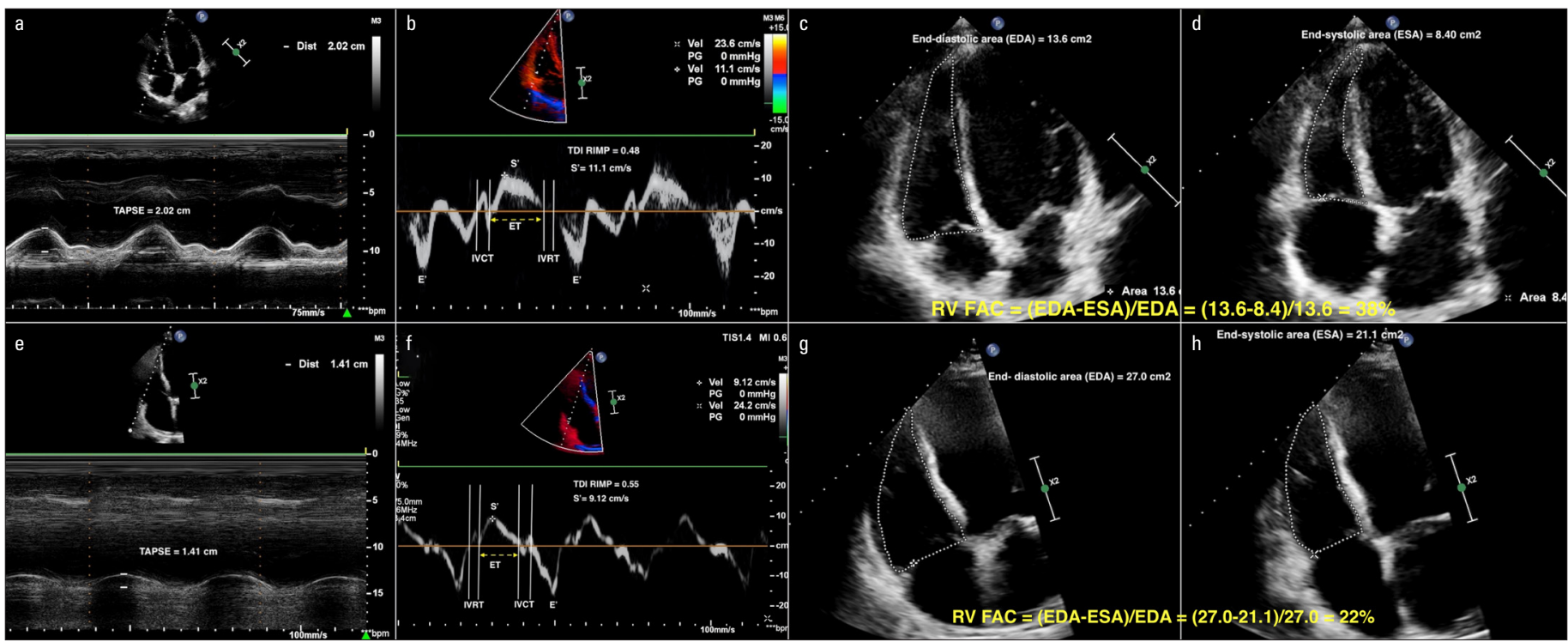

Figure 1. Echocardiographic images of two patients with normal and abnormal right ventricular systolic function parameters. (a) Normal TAPSE (b) Normal RV TDI S' and TDI derived RIMP (c, d) Normal RV FAC (e) Abnormal TAPSE (f) Abnormal RV TDI S' and TDI derived RIMP (g, h) Abnormal RV FAC

RIMP - right ventricular index of myocardial performance; RV- right ventricle; RV FAC - right ventricular fractional area change; TAPSE - tricuspid annular plane systolic excursion; TDI - tissue Doppler imaging

ment provided both for the patient and the echocardiographer. All the images were stored and analyzed at a later time to minimize viral transmission risk. All echocardiographic procedures were compatible with the recommendations of the American Society of Echocardiography $(10,11)$.

Left ventricular end diastolic diameter (LVEDd) was calculated from the parasternal long axis image, and left ventricular ejection fraction (LVEF) was measured using the modified $2 \mathrm{D}$ biplane method of disks summation technique. Left atrium anterior-posterior diameter was calculated from parasternal long axis view at the end of systole. Right atrium (RA) and RV diameters were assessed from apical four-chamber view and RV focused apical four-chamber view. RA end-systolic area was determined by planimetry at the end of ventricular systole, tracing from the lateral aspect of the tricuspid annulus to the septal aspect, excluding the area between the leaflets and annulus.

Mitral inflow $E$ and $A$ waves were measured through left ventricular spectral Doppler analysis. E/A ratio was then calculated. In a similar manner, tricuspid inflow $E$ and $A$ waves and deceleration time were measured. Systolic pulmonary artery pressure (sPAP) was assessed using the modified Bernoulli equation.

Tricuspid annular plane systolic excursion (TAPSE) was calculated through $\mathrm{M}$-mode imaging from apical four-chamber view as the systolic displacement of the tricuspid lateral annulus. TAPSE $<1.7 \mathrm{~cm}$ was defined as RV systolic impairment. Tissue Doppler imaging (TDI) derived tricuspid lateral annular systolic velocity wave $\left(S^{\prime}\right)$ was calculated from $\mathrm{RV}$ focused apical fourchamber view, and $S^{\prime}$ value $<9.5 \mathrm{~cm} / \mathrm{sec}$ was defined as RV systolic impairment. RV fractional area change (FAC) was measured according to the formula: (RV end-diastolic area-RV endsystolic area)/end-diastolic area $\times 100$. RV-FAC $<35 \%$ was defined as RV systolic impairment. RV index of myocardial per- formance (RIMP) was examined from tricuspid lateral annulus through TDI using the formula: (Isovolumic contraction time + isovolumic relaxation time)/ejection time. RIMP value $>0.54$ was defined as RV systolic impairment. Abnormality in any of these parameters including TAPSE, RV TDI derived S', RV FAC, and RV TDI derived RIMP was defined as RV systolic impairment (10, 11). Echocardiographic images of two patients with normal and abnormal RV systolic function parameters are demonstrated in Figure 1.

\section{Data collection and treatment}

Basal demographic and clinical features, comorbidities, previous medications, laboratory parameters, chest CT scans, and echocardiographic data of the patients were obtained in a prospective manner and recorded on a study chart. Clinical status of the patients such as decision of discharge or clinical deterioration was also followed daily and recorded. Medical treatment of patients included hydroxychloroquine, favipravir, antibiotic therapy, and enoxaparin. Treatment decisions were made by the physicians who were blinded to the study, and these physicians assessed the clinical status of the patients daily.

\section{Statistical analyses}

Statistical analyses were carried out using IBM Statistical Package for Social Sciences for Macintosh, version 24.0 (IBM Corp., Armonk, New York, USA). The Shapiro-Wilk test was used to examine the distribution of numerical variables. The chisquared test was applied for categorical variables and presented as percentages. If the chi-squared test could not be applied, Fisher's exact test was used. Student's t-test was applied to the numerical data which conformed to the normal distribution, and the results were entered as mean \pm standard deviation. The 


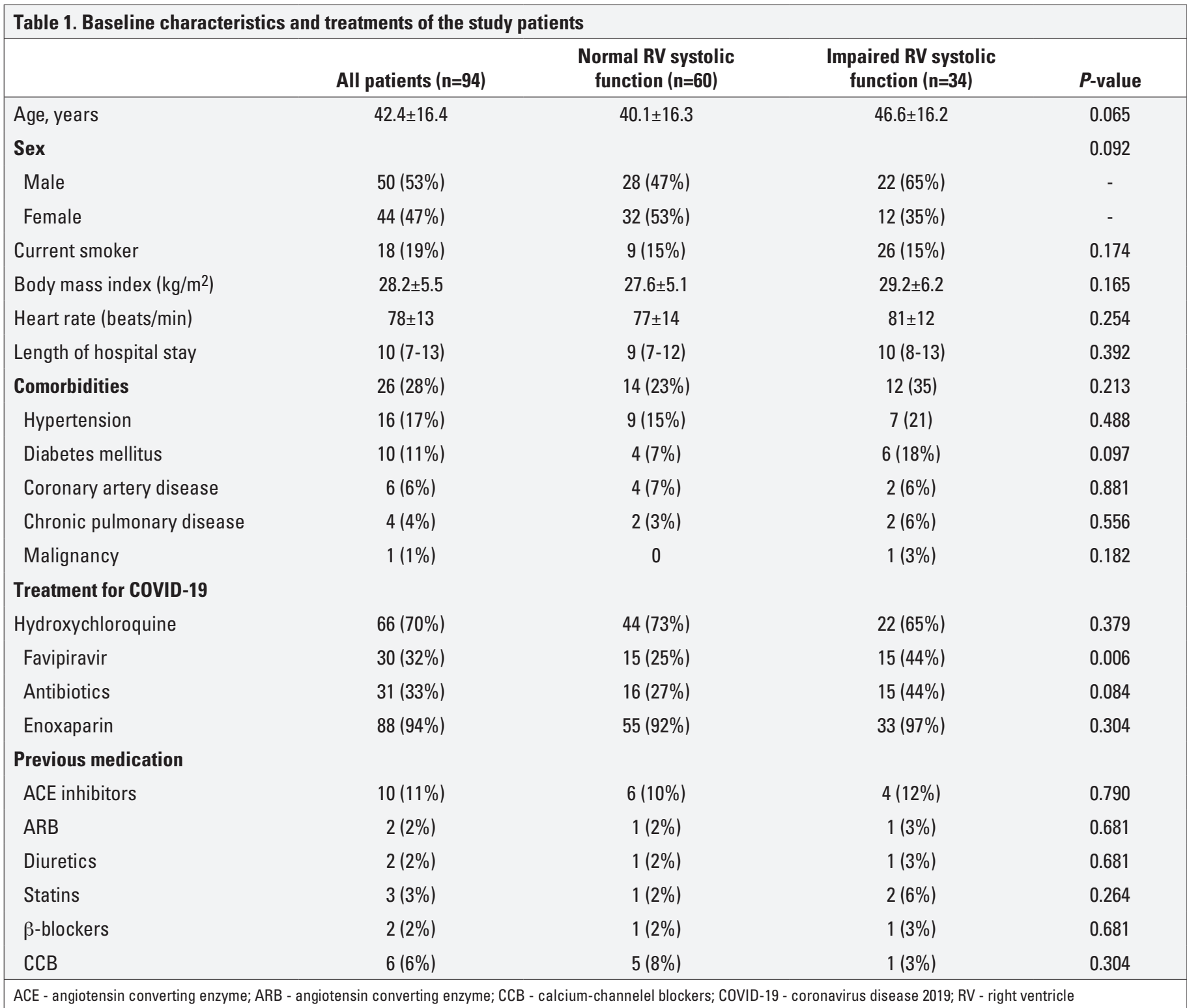

Mann-Whitney $U$ test was applied for the non-normally distributed variables, and the results were given as median with interquartile range. Receiver operating characteristic (ROC) curve analyses were conducted to determine the cut-off values for the sensitivity and specificity of IL-6 for predicting deteriorations in RV systolic function. The area under the ROC curve (AUC) was reported with $95 \%$ confidence interval (CI) in addition to sensitivity, specificity, positive predictive value (PPV), negative predictive value (NPV), positive likelihood ratio, and negative likelihood ratio. Logistic regression analyses were performed to evaluate independent predictors of RV systolic impairment. Variables, which might be a possible confounding factor for RV systolic impairment such as age, lymphocyte count, CRP, ferritin, LVEF, sPAP, abnormal chest CT findings, and IL-6 levels were included in the univariate and multivariate analyses. The goodness-of-fit assumption was examined using the Hosmer-Lemeshow method and satisfied when $p$ value was more than 0.05 . A two-sided alpha value of less than 0.05 was determined to be statistically significant.

\section{Results}

\section{Baseline characteristics and treatments}

A total of 100 consecutive patients were included in the study. After the elimination of six patients per the exclusion criteria, the remaining 94 patients were included in the final analyses. The mean age was $42.4 \pm 16.4$ years, and $53 \%$ of the patients were men. The median length of hospital stay was 10.0 days [interquartile range (IQR) 7.0-13.0]. Comorbidities were present in $28 \%$ of the patients with hypertension (HT) being the most common comorbidity followed by diabetes mellitus (DM) and coronary artery disease (CAD). The patients were classified into two groups according to their RV systolic functions; normal RV systolic functions $(n=60)$ and impaired RV systolic functions ( $n=34$ ) (Table 1). 


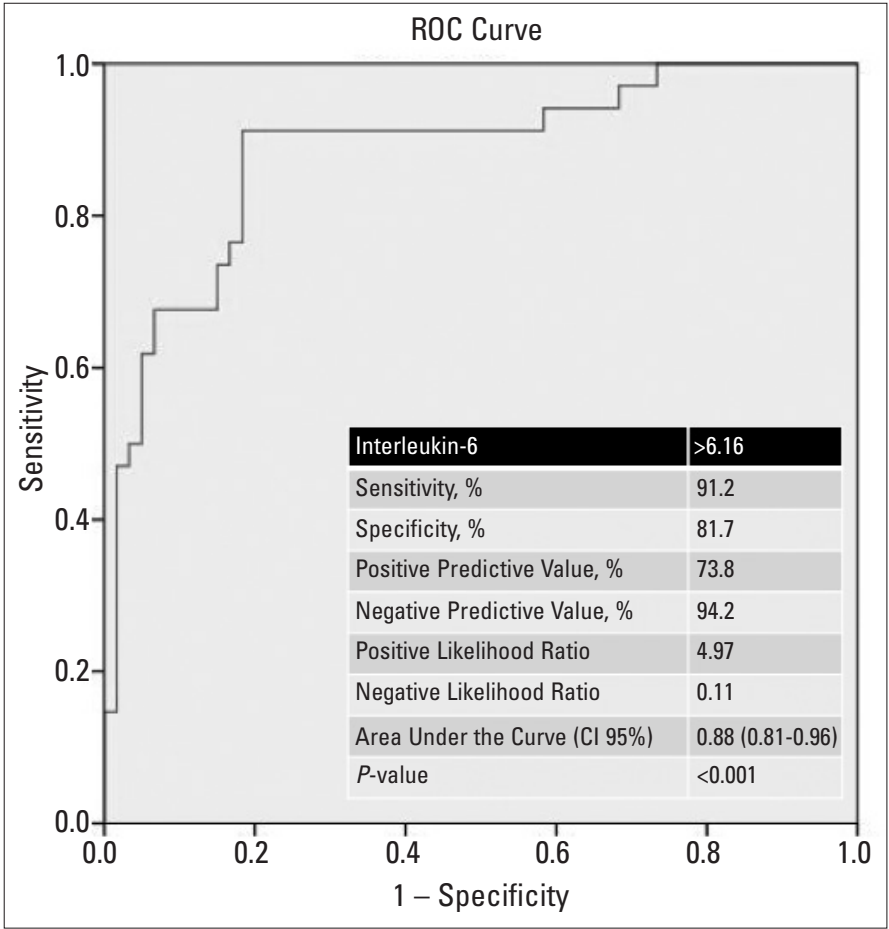

Figure 2. Receiver operating characteristic curve analyses conducted to determine the cut-off values for the sensitivity and specificity of interleukin-6 for predicting right ventricular systolic impairment

There was no difference between the groups in terms of age, sex, smoking status, body mass index, heart rate, length of hospital follow-up, presence of any comorbidity, HT, DM, CAD, chronic pulmonary disease, and malignancy. Previous medications including angiotensin converting enzyme inhibitors, angiotensin receptor blockers, diuretics, statins, beta blockers, and calcium channel blockers were comparable between the two groups. Treatment regimens during the hospital stay were similar, except favipravir (Table 1). According to clinical classification aforementioned in the methodology section, $65 \%$ of the patients $(n=61)$ had moderate COVID-19, and the remaining $35 \%(n=33)$ had mild COVID-19. The percentage of patients with moderate COVID-19 was more in the group with impaired RV systolic functions than in the group with normal RV systolic functions ( $79 \%$ vs. $57 \%, \mathrm{p}=0.026)$.

\section{Laboratory and radiological findings}

Leukocyte counts, neutrophil counts, platelet counts, and hemoglobin levels were similar between the groups, whereas lymphocyte counts were lower in patients with impaired RV systolic function ( $p=0.023)$. The percentage of patients with leukopenia, thrombocytopenia, lymphopenia, and anemia were comparable between the two groups. However, leukocytosis was more prevalent in patients with normal RV systolic functions $(p=0.038)$. There was no difference between the groups in terms of procalcitonin and hs-Tnl levels. D-dimer, CRP, and ferritin levels were significantly increased in patients with impaired RV systolic function than in those with normal RV systolic function ( $p=0.003, p=0.001, p<0.001$, respectively). However, the percentage of patients with negative $D$-dimer values $(\leq 0.5 \mu \mathrm{g} / \mathrm{mL})$ was lower in the group with impaired RV systolic functions $(p<0.001)$. The remaining biochemical parameters, except liver function tests, albumin, sodium, and total cholesterol levels were comparable between the two groups. The median value of IL-6 levels was significantly higher in patients with impaired RV systolic functions than in those with normal RV systolic functions (20.3, 4.6, $\mathrm{p}<0.001$, respectively) (Table 2). The AUC for IL-6 to predict RV systolic impairment was 0.88 (95\% Cl $0.81-0.95$, $p<0.001$ ) with a positive likelihood ratio of 4.97 and negative likelihood ratio of 0.11 . The cut-off value of the IL- 6 level above 6.16 was associated with $91.2 \%$ sensitivity, $81.7 \%$ specificity, PPV $73.8 \%$, and NPV $94.2 \%$ (Fig. 2).

Abnormal chest CT findings were present in $65 \%$ of the study population. Ground-glass opacity was the most common sign followed by consolidation, reticular pattern, and interlobular septal thickening, respectively. The percentage of patients with any abnormal chest CT finding, consolidation, interlobular septal thickening and reticular pattern was significantly increased in the group with impaired RV systolic function than in the group with normal RV systolic function $(p=0.02, p=0.03, p=0.03, p=0.01$, respectively) (Table 2).

\section{Transthoracic echocardiography parameters}

Two-dimensional TTE parameters including LVEDd, LVEF, left atrial diameter, RV basal diameter, RV longitudinal diameter, and RA minor axis diameter were similar between the groups. RA major axis diameter and RA end-systolic area was significantly increased in patients with impaired RV systolic function than in those with normal RV systolic function $(p<0.001, p=0.02$, respectively). There was no difference between the groups in terms of mitral $A$ wave, $E / A$ ratio, tricuspid $E$ wave, and tricuspid deceleration time. However, mitral E wave was lower and SPAP was higher in patients with impaired RV systolic function than in those with normal RV systolic function ( $p=0.01, p=0.02$, respectively) (Table 3 ).

When it comes to RV systolic function, abnormalities in TAPSE, RV TDI derived S', RV FAC, and RV TDI derived RIMP calculations were present in $50 \%, 47 \%, 94 \%$, and $97 \%$ in patients with impaired RV systolic functions, respectively. TAPSE and RV TDI derived $S^{\prime}$ measurements were similar between the two groups. RV FAC was significantly lower in the impaired RV systolic function group than in the normal RV systolic function group (31.9 $\pm 2.7,38.2 \pm 2.7, p<0.001)$. RV TDI derived RIMP was significantly increased in patients with impaired RV systolic functions than in those with normal RV systolic functions $(0.60,0.50$, $\mathrm{p}<0.001$, respectively) (Table 3 ).

\section{Predictors of RV systolic impairment}

Univariate and multivariate regression analyses were performed to evaluate the association between clinical characteristics, laboratory parameters, radiological and echocardiographic findings, and deteriorations in RV systolic function. In the univariate analyses, lymphocytes [odds ratio (OR): $0.35,95 \% \mathrm{Cl}: 0.15-0.79, p=0.01]$, CRP (OR: 1.01, 95\% Cl: $1.00-1.02, p=0.004)$, ferritin (OR: 1.01, 95\% Cl: $1.01-$ 1.02, $p=0.002$ ), IL-6 (OR: 1.12, 95\% Cl: 1.05-1.19, $p=0.01$ ), LVEF (OR: 0.92, $95 \% \mathrm{Cl}: 0.85-0.99, p=0.03$ ), sPAP (OR: 1.07, 95\% Cl: 1.01-1.14, $\mathrm{p}=0.03$ ), 


\begin{tabular}{|c|c|c|c|c|}
\hline & All patients ( $n=94)$ & $\begin{array}{c}\text { Normal RV systolic } \\
\text { function }(n=60)\end{array}$ & $\begin{array}{c}\text { Impaired RV systolic } \\
\text { function }(n=34)\end{array}$ & $P$-value \\
\hline Leucocytes (×109/L) & $5.1(4.0-6.5)$ & $5.1(3.9-6.8)$ & $5.0(4.3-6.3)$ & 0.735 \\
\hline$<4$ & $21(22 \%)$ & $16(27 \%)$ & $5(15 \%)$ & 0.181 \\
\hline $4-10$ & $66(70 \%)$ & $37(62 \%)$ & $29(85 \%)$ & 0.001 \\
\hline$>10$ & $7(11 \%)$ & $7(12 \%)$ & $0(0 \%)$ & 0.038 \\
\hline Lymphocytes (×109/L) & $1.3(1.0-1.9)$ & $1.4(1.0-2.1)$ & $1.3(0.9-1.5)$ & 0.023 \\
\hline$<1.0$ & $20(21 \%)$ & $10(17 \%)$ & $10(29)$ & 0.147 \\
\hline Platelets $\left(\times 10^{9} / \mathrm{L}\right)$ & $237 \pm 65$ & $235 \pm 64$ & $241 \pm 66$ & 0.662 \\
\hline$<150$ & $7(7 \%)$ & $5(8 \%)$ & $2(6 \%)$ & 0.664 \\
\hline Hemoglobin (g/L) & $13.5 \pm 1.6$ & $13.5 \pm 1.6$ & $13.5 \pm 1.8$ & 0.948 \\
\hline$>1$ & $18(19 \%)$ & $10(17 \%)$ & $8(23 \%)$ & 0.416 \\
\hline C-reactive protein $(\mathrm{mg} / \mathrm{L})$ & $6.9(3.0-26.2)$ & $4.6(2.0-15.2)$ & $23.5(5.0-56.5)$ & 0.001 \\
\hline$<5$ & $39(41 \%)$ & $32(53 \%)$ & $7(21)$ & 0.002 \\
\hline$\geq 5$ & $55(59 \%)$ & $28(47 \%)$ & $27(79 \%)$ & - \\
\hline Ferritin $(\mu \mathrm{g} / \mathrm{L})$ & $123(43-226)$ & $59(23-128)$ & $227(179-314)$ & $<0.001$ \\
\hline$<300$ & $80(85 \%)$ & $55(92 \%)$ & $25(73 \%)$ & 0.018 \\
\hline$\geq 300$ & $14(15 \%)$ & $5(8 \%)$ & $9(26 \%)$ & - \\
\hline Interleukin-6 (pg/mL) & $6.4(3.8-20.0)$ & $4.6(3.6-6.4)$ & $20.3(7.7-36.7)$ & $<0.001$ \\
\hline hs-Tnl (ng/L) & $2.5(2.5-6.0)$ & $2.5(2.5-4.0)$ & $3.5(2.5-6.2)$ & 0.097 \\
\hline $\operatorname{ALT}(\mathrm{U} / \mathrm{L})$ & $29(19-38)$ & $27(17-36)$ & $31(23-52)$ & 0.032 \\
\hline Total cholesterol (mg/dL) & $153 \pm 31$ & $158 \pm 32$ & $143 \pm 26$ & 0.038 \\
\hline Triglycerides (mg/dL) & $120 \pm 57$ & $121 \pm 59$ & $118 \pm 55$ & 0.832 \\
\hline $\mathrm{HDL}(\mathrm{mg} / \mathrm{dL})$ & $37(31-44)$ & $38(32-45)$ & $34(29-41)$ & 0.173 \\
\hline $\operatorname{LDL}(\mathrm{mg} / \mathrm{dL})$ & $91 \pm 28$ & $94 \pm 29$ & $85 \pm 24$ & 0.118 \\
\hline Abnormal chest CT & $61(65 \%)$ & $34(57 \%)$ & $27(79 \%)$ & 0.026 \\
\hline Ground-glass opacity & $58(62 \%)$ & $34(57 \%)$ & $24(70 \%)$ & 0.182 \\
\hline Consolidation & $17(18 \%)$ & $7(12 \%)$ & $10(29 \%)$ & 0.032 \\
\hline Interlobular septal thickening & $5(5 \%)$ & $1(2 \%)$ & $4(12 \%)$ & 0.036 \\
\hline Reticular pattern & $6(6 \%)$ & $1(2 \%)$ & $5(15 \%)$ & 0.013 \\
\hline
\end{tabular}

and abnormal chest CT findings (OR: 2.95, 95\% Cl: 1.11-7.82, $\mathrm{p}=0.03$ ) were related to RV systolic impairment. Multivariate regression analysis was then performed to detect independent predictors of RV systolic impairment. However, only IL-6 levels independently predicted RV systolic impairment at a significant level (OR: 1.12, 95\% Cl: 1.04-1.20, p=0.003) (Table 4, Supplement Table 1).

\section{Discussion}

In this study, we aimed to examine whether increased IL-6 levels can predict RV systolic impairment in patients with COVID-19. According to our knowledge, this is the first study in the literature evaluating the relationship between RV functions 


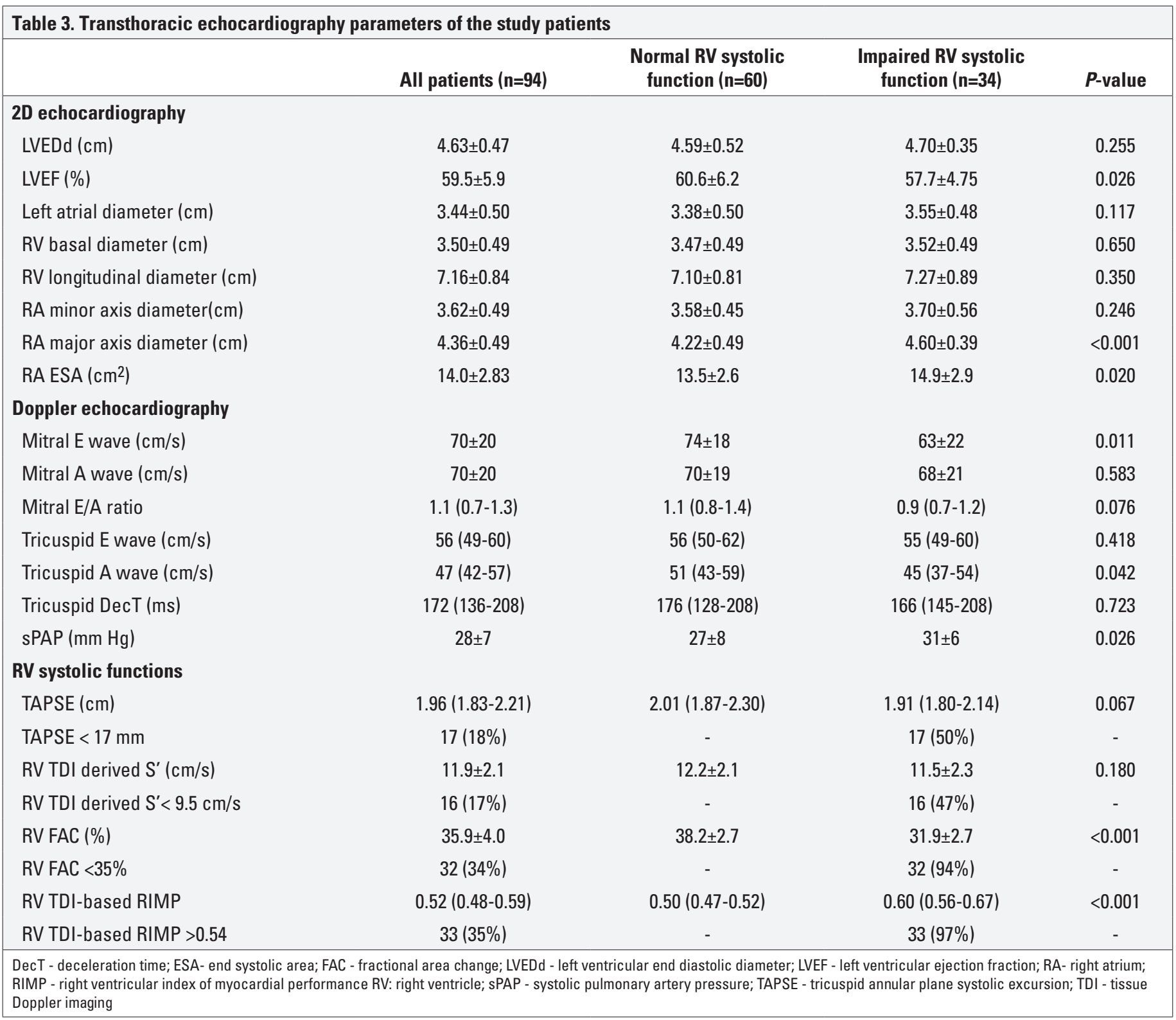

\begin{tabular}{|c|c|c|c|c|}
\hline & \multicolumn{2}{|c|}{ Univariate } & \multicolumn{2}{|c|}{ Multivariate*,† } \\
\hline & OR (95\% CI) & $P$-value & OR (95\% Cl) & $P$-value \\
\hline Lymphocytes & $0.35(0.15-0.79)$ & 0.011 & $0.40(0.13-1.28)$ & 0.122 \\
\hline C-reactive protein & $1.01(1.00-1.02)$ & 0.004 & $1.01(0.99-1.01)$ & 0.819 \\
\hline LVEF & $0.92(0.85-0.99)$ & 0.030 & $0.91(0.82-1.02)$ & 0.114 \\
\hline sPAP & $1.07(1.01-1.14)$ & 0.031 & $1.04(0.95-1.13)$ & 0.398 \\
\hline Abnormal chest CT findings & $2.95(1.11-7.82)$ & 0.030 & $1.01(0.27-3.8)$ & 0.994 \\
\hline \multicolumn{5}{|c|}{$\begin{array}{l}\text { *Nagelkerke } \mathrm{R} \text { square }=0.522 ;-2 \text { log likelihood }=77.9 ; \quad P \text {-value }<0.001 \\
\text { †Hosmer-Lemeshow test's chi-squared value }=8.7 ; \quad P \text {-value }=0.368 \\
P \text {-value less than } 0.05 \text { was considered significant for statistical analyses } \\
\text { Cl - confidence interval; CT - computed tomography; LVEF - left ventricular ejection fraction; OR - odds ratio; } \text { SPAP - systolic pulmonary artery pressure }\end{array}$} \\
\hline
\end{tabular}


and IL-6 levels in patients with COVID-19. Our analyses demonstrated that increased IL-6 levels independently predict RV systolic impairment in this specific patient group.

Systemic inflammatory response activated by the binding of SARS-CoV-2 to alveolar epithelium with resulting innate and adaptive immune system alterations and the subsequent cytokine activation are the main pathophysiological processes of SARS-CoV-2 infection (12). Cytokine release, which is also called a cytokine storm, is strongly associated with unwanted outcomes and disease severity in these patients $(13,14)$. Among many inflammatory system cells and cytokines, IL- 6 has been suggested to be at the forefront of the COVID-19 pathophysiology. In addition to its role as an acute phase reactant, IL-6 also plays a role in hematopoiesis, metabolic regulation, and autoimmunity (12). From a clinical perspective, increased IL-6 levels were shown to predict poor prognosis in patients with COVID-19 $(1,15)$. Tocilizumab, which is an IL-6 receptor antagonist, has been suggested as a possible therapeutic agent for the treatment of COVID-19 pneumonia (16).

The close relationship between IL- 6 and cardiovascular system is well-known. Higher plasma IL-6 levels were found to be related with larger infarct size and decreased cardiac function in patients with CAD in a recent study (17). Besides, IL-6 level was shown to be an independent predictor of RV dilation detected through magnetic resonance imaging (MRI) in asymptomatic individuals free of cardiovascular disease (6). In addition, a TTE based study demonstrated that serum IL-6 levels are independently associated with RV function and in patients with RV-pulmonary artery coupling in pulmonary artery HT (7). According to the previously published literature, we have hypothesized that IL-6 levels are associated with RV functions in patients with COVID-19. In addition to IL-6, diverse molecules, biomarkers, and immune system cells were tested in the univariate analysis of the study, such as CRP, ferritin, and lymphocyte cell counts. All these variables were associated with deterioration in RV systolic function, but only IL-6 levels emerged as an independent predictor of RV systolic impairment in patients with COVID-19 in the multivariate analysis.

TTE, which is a non-invasive and reproducible imaging method, allows for the quantitative and qualitative assessment of cardiac anatomy and functions. It is also an invaluable tool to evaluate RV function in daily clinical practice $(18,19)$. Although cardiac MRI is the gold standard for determining RV function, the time needed for adequate examination, contrast media reaction, relatively high cost, and breath-holds during the procedure are major issues restricting its use $(20,21)$. Difficulty of managing risk for viral contagion and patients for holding breath made us to choose $2 \mathrm{D}$ echocardiography to evaluate RV function in this study. However, deteriorations in RV function or morphology detected through TTE in patients with COVID-19 were shown in recent studies. For example, RV dilation was significantly associated with in-hospital mortality in patients hospitalized with COVID-19 (22). Similarly, RV longitudinal strain impairment was found to be an independent predictor of poor outcomes, including mortality in patients infected with SARS-CoV-2
(23). RV dilation and dysfunction were prevalent in patients with COVID-19, and RV systolic dysfunction was linked to increased D-dimer and CRP levels in another study. In addition, there were hyperdynamic or normal left ventricular function in most patients (24). LV function was also at normal level in our study population. At this point, it is a reasonable question why RV and not LV systolic functions were impaired and associated with IL-6 levels in the study group. It is known that pneumonia affects primarily RV functions rather than LV, which might be owing to increased pulmonary artery pressure, RV afterload, acidosis, and global hypoxemia. It should also be emphasized that because we aimed to investigate RV systolic function, LVEF measurements were restricted to modified $2 \mathrm{D}$ biplane method of disks summation technique instead of detailed and more sensitive modalities such as cardiac MRI, LV strain, speckle tracking, and/or 3D imaging.

The patients included in our study were in relatively better clinical condition in terms of SARS-CoV-2 infection. We did not include patients with invasive mechanical ventilation or patients with severe condition for several reasons. First, it might have been difficult to obtain well-qualified images in intubated patients. Second, invasive mechanical ventilation itself might be associated with RV systolic impairment as demonstrated in a previous study (25). Third, prone positioning, a widely used treatment in patients with ARDS, can make the evaluation of RV function problematic (26). Although the clinical status of some patients worsened during the hospital follow-up, we did not exclude them from the final analysis because of the observational nature of the study. Our study results also suggest that there is a global systolic deterioration in RV function in COVID-19 as demonstrated by impaired RV TDI, RIMP, and FAC values in contrast to normal longitudinal systolic functions such as TAPSE and RV TDI derived $S^{\prime}$ values. Furthermore, our study puts forth pathogenetic clues for impairment of RV systolic function unlike other TTE based studies. In our study, IL-6 emerged as an independent predictor of RV systolic impairment in patients with COVID-19 suggesting a potential pathophysiological role.

The role of cardiac imaging in our daily practice has evolved during this pandemic. Although routine and/or elective echocardiographic evaluation is not recommended because of viral transmission risk and labor saving (27), echocardiographic findings might have a beneficial role during the decision making process in appropriate clinical settings such as diagnosis of left ventricular dysfunction and/or myocarditis (28). Biomarkerbased strategies might also give beneficial clues during this process. The most evident example of this strategy comes from a recent study demonstrating that patients with severe COVID19 and elevated D-dimer levels (>6 times the upper limit of normal values) are likely to benefit from heparin treatment (29). In a similar manner, peak cardiac $\mathrm{Tnl}$ but not admission $\mathrm{Tnl}$ levels were shown to predict death within a one week period in patients with COVID-19 (30). Our study was not designed to predict mortality; but on the basis of previous studies and our study, it can be speculated that biomarker-based strategies may help to predict adverse outcomes, such as deterioration in RV sys- 
tolic function as investigated in our study, in patients suffering from COVID-19. For instance, combining myocardial injury marker troponin with abnormal TTE findings predicted in-hospital mortality in patients with COVID-19 according to a recent study (31).

\section{Study limitations}

Although our data indicated a predictive role for IL- 6 in the deterioration of RV systolic function in patients with COVID-19, there are many questions and limitations that need to be tested in future studies. First, the small size of the study population and lack of a control group without COVID-19 impairs the robustness of the findings. Furthermore, this study included patients in relatively stable clinical conditions compared with other hospitalized patients such as patients on mechanical ventilation and/or in ICUs. It should also be indicated that our study group included relatively younger patients, and thus, there is a need for confirmation of these results in elderly patients. Nevertheless, independent association of IL-6 and RV systolic impairment even in a younger and less critical patient population emphasizes the significance of IL-6 as a biomarker to predict RV systolic function in COVID-19. It is also possible that there may be patients with COVID-19 not referred for echocardiographic examination. It should also be noted that MRI examination is superior to TTE for evaluating RV function as discussed above. Superiority of 3D TTE to 2D echocardiographic examination and lack of strain imaging data in this study should also be considered. The observational nature of our study also limited testing the prognostic role of IL-6 and RV systolic impairment in COVID-19. Follow-up and temporal trends of IL-6 levels could yield more beneficial evidence.

\section{Conclusion}

IL-6 is an independent predictor of RV systolic impairment in patients hospitalized with mild and moderate COVID-19 suggesting a possible pathogenetic mechanism. IL-6 levels can be used to predict RV systolic impairment in patients suffering from this infection.

Conflict of interest: None declared.

Peer-review: Externally peer-reviewed.

Author contributions: Concept - M.E.; Design - M.E., M.A.E.; Supervision - R.G.; Fundings - None; Materials - A.K.K., B.K.; Data collection \&/or processing - M.E., S.Ö., Y.E.Ö.; Analysis \&/or interpretation - M.E.; Literature search - M.E.; Writing - S.Ö.; Critical review - R.G.

\section{References}

1. Zhou F, Yu T, Du R, Fan G, Liu Y, Liu Z, et al. Clinical course and risk factors for mortality of adult inpatients with COVID-19 in Wuhan, China: a retrospective cohort study. Lancet 2020; 395: 1054-62. [Crossref]

2. Cummings MJ, Baldwin MR, Abrams D, Jacobson SD, Meyer BJ, Balough EM, et al. Epidemiology, clinical course, and outcomes of critically ill adults with COVID-19 in New York City: a prospective cohort study. Lancet 2020; 395: 1763-70. [Crossref]

3. Zangrillo A, Beretta L, Scandroglio AM, Monti G, Fominskiy E, Colombo S, et al.; COVID-BioB Study Group. Characteristics, treatment, outcomes and cause of death of invasively ventilated patients with COVID-19 ARDS in Milan, Italy. Crit Care Resusc 2020 Apr 23. [Epub ahead of print]

4. Repessé X, Vieillard-Baron A. Right heart function during acute respiratory distress syndrome. Ann Transl Med 2017; 5: 295. [Crossref]

5. Chen N, Zhou M, Dong X, Qu J, Gong F, Han Y, et al. Epidemiological and clinical characteristics of 99 cases of 2019 novel coronavirus pneumonia in Wuhan, China: a descriptive study. Lancet 2020; 395: 507-13. [Crossref]

6. Harhay MO, Tracy RP, Bagiella E, Barr RG, Pinder D, Hundley WG, et al. Relationship of CRP, IL-6, and fibrinogen with right ventricular structure and function: the MESA-Right Ventricle Study. Int J Cardiol 2013; 168: 3818-24. [Crossref]

7. Prins KW, Archer SL, Pritzker M, Rose L, Weir EK, Sharma A, et al. Interleukin-6 is independently associated with right ventricular function in pulmonary arterial hypertension. J Heart Lung Transplant 2018; 37: 376-84. [Crossref]

8. World Health Organization. Clinical management of severe acute respiratory infection when novel coronavirus (2019-nCoV) infection is suspected: interim guidance, 28 January 2020. World Health Organization (2020). Available from; URL: https://apps.who.int/iris/ handle/10665/330893

9. Diagnosis and Treatment Protocol for Novel Coronavirus Pneumonia (Trial Version 7). Chin Med J (Engl) 2020; 133: 1087-95. [Crossref]

10. Rudski LG, Lai WW, Afilalo J, Hua L, Handschumacher MD, Chandrasekaran K, et al. Guidelines for the echocardiographic assessment of the right heart in adults: a report from the American Society of Echocardiography endorsed by the European Association of Echocardiography, a registered branch of the European Society of Cardiology, and the Canadian Society of Echocardiography. J Am Soc Echocardiogr 2010; 23: 685-713. [Crossref]

11. Lang RM, Badano LP, Mor-Avi V, Afilalo J, Armstrong A, Ernande L, et al. Recommendations for cardiac chamber quantification by echocardiography in adults: an update from the American Society of Echocardiography and the European Association of Cardiovascular Imaging. J Am Soc Echocardiogr 2015; 28: 1-39.e14. [Crossref]

12. Gubernatorova E0, Gorshkova EA, Polinova Al, Drutskaya MS. IL-6: Relevance for immunopathology of SARS-CoV-2. Cytokine Growth Factor Rev 2020; 53: 13-24. [Crossref]

13. Huang C, Wang Y, Li X, Ren L, Zhao J, Hu Y, et al. Clinical features of patients infected with 2019 novel coronavirus in Wuhan, China. Lancet 2020; 395: 497-506. [Crossref]

14. Liu J, Li S, Liu J, Liang B, Wang X, Wang H, et al. Longitudinal characteristics of lymphocyte responses and cytokine profiles in the peripheral blood of SARS-CoV-2 infected patients. EBioMedicine 2020; 55: 102763. [Crossref]

15. Gao Y, Li T, Han M, Li X, Wu D, Xu Y, et al. Diagnostic utility of clinical laboratory data determinations for patients with the severe COVID19. J Med Virol 2020; 92: 791-6. [Crossref]

16. Zhang S, Li L, Shen A, Chen Y, Qi Z. Rational Use of Tocilizumab in the Treatment of Novel Coronavirus Pneumonia. Clin Drug Investig 2020; 40: 511-8. [Crossref] 
17. Groot $\mathrm{HE}$, Al Ali $\mathrm{L}$, van der Horst ICC, Schurer RAJ, van der Werf HW, Lipsic E, et al. Plasma interleukin 6 levels are associated with cardiac function after ST-elevation myocardial infarction. Clin Res Cardiol 2019; 108: 612-21. [Crossref]

18. Schneider M, Binder T. Echocardiographic evaluation of the right heart. Wien Klin Wochenschr 2018; 130: 413-20. [Crossref]

19. Hillis GS, Bloomfield P. Basic transthoracic echocardiography. BMJ 2005; 330: 1432-6. [Crossref]

20. D'Ascenzi F, Anselmi F, Piu P, Fiorentini C, Carbone SF, Volterrani L, et al. Cardiac Magnetic Resonance Normal Reference Values of Biventricular Size and Function in Male Athlete's Heart. JACC Cardiovasc Imaging 2019; 12: 1755-65. [Crossref]

21. Saeed M, Van TA, Krug R, Hetts SW, Wilson MW. Cardiac MR imaging: current status and future direction. Cardiovasc Diagn Ther 2015; 5: 290-310.

22. Argulian E, Sud K, Vogel B, Bohra C, Garg VP, Talebi S, et al. Right Ventricular Dilation in Hospitalized Patients With COVID-19 Infection. JACC Cardiovasc Imaging 2020; 13: 2459-61. [Crossref]

23. Li Y, Li H, Zhu S, Xie Y, Wang B, He L, et al. Prognostic Value of Right Ventricular Longitudinal Strain in Patients With COVID-19. JACC Cardiovasc Imaging 2020; 13: 2287-99. [Crossref]

24. Mahmoud-Elsayed HM, Moody WE, Bradlow WM, Khan-Kheil AM, Senior J, Hudsmith LE, et al. Echocardiographic Findings in Patients With COVID-19 Pneumonia. Can J Cardiol 2020; 36: 1203-7. [Crossref]
25. Rana M, Yusuff H, Zochios V. The Right Ventricle During Selective Lung Ventilation for Thoracic Surgery. J Cardiothorac Vasc Anesth 2019; 33: 2007-16. [Crossref]

26. Giustiniano E, Padua E, Negri K, Bragato RM, Cecconi M. Echocardiography during Prone-Position Mechanical Ventilation in Patients with COVID-19: A Proposal for a New Approach. J Am Soc Echocardiogr 2020; 33: 905-6. [Crossref]

27. Skulstad H, Cosyns B, Popescu BA, Galderisi M, Salvo GD, Donal E, et al. COVID-19 pandemic and cardiac imaging: EACVI recommendations on precautions, indications, prioritization, and protection for patients and healthcare personnel. Eur Heart $J$ Cardiovasc Imaging 2020; 21: 592-8. [Crossref]

28. Kim IC, Kim JY, Kim HA, Han S. COVID-19-related myocarditis in a 21-year-old female patient. Eur Heart J 2020; 41: 1859. [Crossref]

29. Tang N, Bai H, Chen X, Gong J, Li D, Sun Z. Anticoagulant treatment is associated with decreased mortality in severe coronavirus disease 2019 patients with coagulopathy. J Thromb Haemost 2020; 18: 1094-9. [Crossref]

30. Deng Q, Hu B, Zhang Y, Wang H, Zhou X, Hu W, et al. Suspected myocardial injury in patients with COVID-19: Evidence from frontline clinical observation in Wuhan, China. Int J Cardiol 2020; 311: 116-21. [Crossref]

31. Giustino G, Croft LB, Stefanini GG, Bragato R, Silbiger JJ, Vicenzi M, et al. Characterization of Myocardial Injury in Patients With COVID-19. J Am Coll Cardiol 2020; 76: 2043-55. [Crossref] 
Supplement Table 1. Association between IL-6 levels and right ventricular systolic function parameters in the whole study population

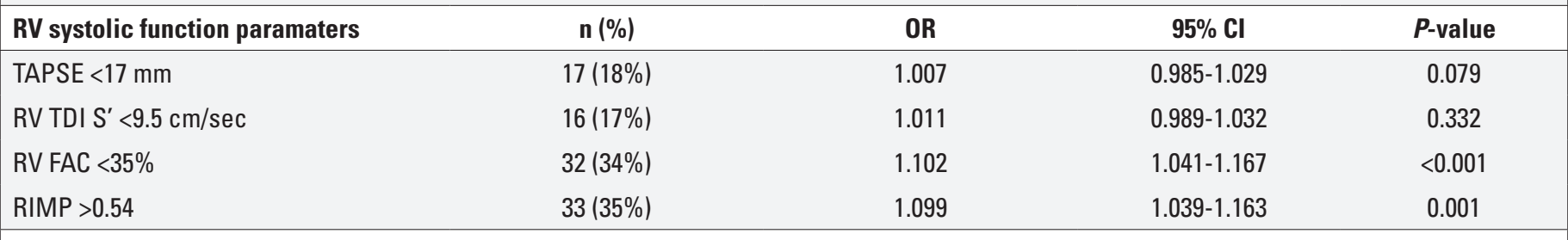

Adjusted to abnormal chest CT findings, LVEF and CRP levels

$\mathrm{Cl}$ - confidence interval; OR - odds ratio; RIMP - right ventricular index of myocardial performance; RV - right ventricle; RV FAC - right ventricular fractional area change; TAPSE tricuspid annular plane systolic excursion; TDI - tissue Doppler imaging; CT - computed tomography; LVEF - left ventricular ejection fraction; CRP - C-reactive protein 